\title{
Robotic versus standard laparoscopic total mesorectal excision for rectal cancer: a comparative study of short-term and oncological outcomes
}

\author{
This article was published in the following Dove Press journal: \\ Robotic Surgery: Research and Reviews \\ 5 September 2014 \\ Number of times this article has been viewed
}

\author{
Jarrod KH Tan' \\ Frederick HX Koh ${ }^{2}$ \\ Charles BS Tsang ${ }^{1,3,4}$ \\ Dean Chi-Siong Koh ${ }^{1,3,4}$ \\ 'Department of Surgery, Yong Loo \\ Lin School of Medicine, National \\ University of Singapore, Singapore; \\ ${ }^{2}$ Department of Surgery, National \\ University Health System, Singapore; \\ ${ }^{3}$ Division of Surgical Oncology, \\ National University Cancer Institute, \\ Singapore; ${ }^{4}$ Colorectal Clinic \\ Associates, Mount Elizabeth Novena \\ Hospital, Singapore
}

\begin{abstract}
Introduction: Laparoscopic total mesorectal excision (TME) is the standard operation for minimal access surgical treatment for rectal cancers. The superiority of the different laparoscopic modalities used to perform TME remains controversial. This study aims to compare the short-term outcomes between robotic TME (R-TME) and the standard laparoscopic TME (ST-TME).
\end{abstract}

Methods: A retrospective review of all patients $(n=42)$ diagnosed with mid/lower rectal carcinoma who underwent R-TME and ST-TME from October 2004 to November 2011 was performed. Patient demographics, perioperative outcomes, and histopathological findings were analyzed.

Results: There were 23 patients treated with R-TME. Patient demographics were comparable between both groups. The median operating time was 242 (191-377) minutes in ST-TME and 395 (289-771) minutes in R-TME $(P<0.001)$. ST-TME was associated with a higher conversion rate, at $21.0 \%$, compared to the $4.3 \%$ in R-TME $(P=0.158)$. Both groups had a median duration of intravenous analgesia of 2 days $(P=0.602)$, and a median length of hospital stay of 6 (ST-TME) and 7 days (R-TME) $(P=0.202)$. Morbidity rates were $31.6 \%$ and $21.7 \%$ in the ST-TME and R-TME groups, respectively $(P=0.504)$. The median number of lymph nodes harvested was the same in each group, at $14(P=0.323)$. Completeness of TME and margin positivity were similar for both groups.

Conclusion: R-TME is safe and feasible, with similar postoperative and surrogate oncological outcomes when compared to ST-TME. While associated with a lower conversion rate, the longer operating time reflects the steep learning curve required to master the technique.

Keywords: robotic, standard laparoscopic, total mesorectal excision, rectal cancer, Da Vinci, rectal cancer, laparoscopy, proctectomy

\section{Introduction}

Since the first laparoscopic colorectal surgery was described in the $1990 \mathrm{~s},{ }^{1}$ minimally invasive surgery for colorectal cancers has gained popularity and acceptance by many. A number of studies have successfully proven that, compared to open surgeries, laparoscopic colonic resections result in decreased postsurgical pain, better cosmesis, and shorter hospital stays while maintaining comparable oncological clearance and morbidity rates. ${ }^{2-4}$

The results presented in the various studies, however, mask the potentially less than favorable outcomes of laparoscopic rectal cancers given that the gold standard treatment
Correspondence: Dean Chi-Siong Koh Colorectal Clinic Associates, Mount Elizabeth Novena Hospital, 38 Irrawaddy Road, \#10-48/49, Singapore 329563 Tel +6581812302

Email drdeankoh@colorectalclinic.com 
for rectal cancers requires access deep into the pelvis to achieve a total mesorectal excision (TME). ${ }^{5-10}$ While proven to be technically feasible, the rigid laparoscopic instruments make the procedure an extremely demanding one. Indeed, the Medical Research Council (MRC) CLASICC trial has demonstrated higher conversion rates and circumferential margin positivity in laparoscopic proctectomies compared to those of laparoscopic colectomies, albeit with comparable oncological clearance and rates of local recurrences. ${ }^{2}$

With the advent of robotic surgery, many postulate that the advantages of three-dimensional visualization, a more stable camera platform, better ergonomics, and increased flexibility of the instruments present can overcome the difficulties faced in laparoscopic rectal resections. ${ }^{11-13}$ Robotic surgery has since been incorporated into rectal cancer treatments in many centers in the world, and a number of studies have been conducted that compare the outcomes between both procedures. Results remain, however, inconclusive, ${ }^{14-19}$ and the two multicenter, randomized controlled trials, RObotic Versus LAparoscopic Resection for Rectal Cancer (ROLARR) and ACOSOG (American College of Surgeons Oncology Group; ACOSOG-Z6051) are still ongoing. ${ }^{20,21}$ This study, therefore, aims to further substantiate the comparison between robotic TME (R-TME) and standard laparoscopic TME (ST-TME) for rectal cancers, as well as provide local data that have yet to be reported.

\section{Materials and methods}

A retrospective review of all patients who underwent R-TME and ST-TME for rectal cancers from October 2004 to November 2011 (Department of Surgery, National University of Singapore) was performed.

The local staging of the rectal tumors was carried out using either endorectal ultrasonography, magnetic resonance imaging of the rectum, or both. The computed tomographic scan of the abdomen and pelvis was performed routinely to establish the presence of systemic metastases. All cases were subjected to a multidisciplinary board meeting held weekly to decide upon the management strategy of the patients. The type of TME performed, whether R-TME or ST-TME, was based on the preference of individual surgeons. Surgeons involved in these operations were at least consultant-grade colorectal surgeons who had each performed in excess of 300 laparoscopic colorectal resections.

Patient demographics collected include age; sex; American Society of Anesthesiologists (ASA) score; number of previous abdominal surgeries; neoadjuvant chemoradiotherapy treatment; and comorbidities, such as hypertension, dyslipidemia, diabetes mellitus, and ischemic heart disease, were collected. Outcome parameters were the types of surgical procedure, operative times, conversion rates, perioperative complications, duration of intravenous opioid usage, and postoperative hospital stays. Histopathological assessment in the form of completeness of TME, distal and circumferential margin tumor involvement, tumor stage, and the total number of lymph nodes harvested were also retrieved.

The standard da Vinci ${ }^{\circledR}$ Surgical System (Intuitive Surgical, Inc., Sunnyvale, CA, USA) was used from August 2008 to May 2010; the newer da Vinci $\mathrm{Si}^{\circledR}$ (Intuitive Surgical, Inc.) was utilized subsequently. Conversion was defined as the need to create an incision which was longer than originally planned in order to perform any part of the operation other than specimen extraction or anastomosis creation. ${ }^{22}$ The cancers were staged according to the American Joint Committee of Cancer guidelines, while complications were graded in accordance to the classification proposed by Clavien et al. ${ }^{23}$

Categorical variables were compared using the Fisher's exact test, while continuous variables were compared using the Mann-Whitney $U$ test. Statistical analysis was performed using the SPSS statistical package (v 19.0; IBM Corporation, Armonk, NY, USA), and all $P$-values reported were two-sided, with $P$-values of $<0.05$ considered statistically significant.

Table I Patient demographics

\begin{tabular}{|c|c|c|c|}
\hline & ST-TME & R-TME & $P$-value \\
\hline Total number of patients & 19 & 23 & \\
\hline Median age, years (range) & $56(42-75)$ & $59(47-92)$ & 0.255 \\
\hline \multicolumn{4}{|l|}{ Sex, n (\%) } \\
\hline Male & II (57.9) & $18(78.3)$ & 0.192 \\
\hline Female & $8(42.1)$ & $5(21.7)$ & \\
\hline \multicolumn{4}{|c|}{ Number of previous abdominal surgeries, $n(\%)$} \\
\hline 0 & $16(84.2)$ & $17(73.9)$ & 0.477 \\
\hline $\mathrm{I}-2$ & $3(15.8)$ & $6(26.1)$ & \\
\hline \multicolumn{4}{|l|}{ Comorbidity, n (\%) } \\
\hline Hypertension & $6(31.6)$ & II (47.8) & 0.353 \\
\hline Dyslipidemia & $3(15.6)$ & $5(21.7)$ & 0.709 \\
\hline Diabetes & $\mathrm{I}(5.3)$ & $4(17.4)$ & 0.356 \\
\hline Ischemic heart disease & $0(0.0)$ & $2(8.7)$ & 0.492 \\
\hline Neoadjuvant & $6(31.6)$ & II (47.8) & 0.353 \\
\hline \multicolumn{4}{|l|}{ chemoradiotherapy, n (\%) } \\
\hline \multicolumn{4}{|l|}{ Type of procedure, n (\%) } \\
\hline Low anterior resection & $17(89.5)$ & $19(82.6)$ & 0.673 \\
\hline Abdominoperineal resection & $2(10.5)$ & $4(17.4)$ & \\
\hline \multicolumn{4}{|l|}{ ASA score, n (\%) } \\
\hline 1 & II (57.9) & $6(26.1)$ & 0.059 \\
\hline ॥ & $8(42.1)$ & $16(69.6)$ & \\
\hline III & $0(0.0)$ & I (4.3) & \\
\hline $\begin{array}{l}\text { Median distance from anal } \\
\text { verge, } \mathrm{cm} \text { (range) }\end{array}$ & $6.0(4-10)$ & $5.0(I-8)$ & 0.119 \\
\hline
\end{tabular}

Abbreviations: ASA, American Society of Anesthesiologists; R-TME, robotic total mesorectal excision; ST-TME, standard laparoscopic total mesorectal excision. 


\section{Results}

Table 1 presents the demographics of the patients selected. The median age of the 19 ST-TME patients was 56 years, and that of the 23 R-TME patients was 59 years $(P=0.255)$. The percentages of male patients were $57.9 \%(\mathrm{n}=11)$ and $78.3 \%(\mathrm{n}=78.3)$ in the ST-TME and R-TME patient groups, respectively $(P=0.192)$. The majority of the patients had not undergone any previous abdominal surgeries, with only $15.8 \%$ (ST-TME) and $26.1 \%$ (R-TME) of the patients having done so $(P=0.477)$. Patient comorbidities such as hypertension, dyslipidemia, diabetes, and ischemic heart disease were not significantly different between both groups. More patients (47.8\%) in the R-TME group had received neoadjuvant chemoradiotherapy, compared to the $31.6 \%$ in the ST-TME group, but this was not found to be statistically significant $(P=0.353)$. There were four $(17.4 \%)$ patients in the robotic arm and two (10.5\%) in the straight laparoscopic arm who underwent an abdominal perineal resection, while the rest of the patients had a low anterior resection performed. All patients within the study had a temporary diverting stoma created, except for the six patients who underwent an abdominoperineal resection (APR). These patients received permanent colostomies. Most patients in both groups had an ASA score of either 1 or 2 , with only one (4.3\%) patient in the R-TME group having an ASA score of III $(P=0.059)$. In addition, the median distance from the distal end of the tumor to the anal verge was shorter in the R-TME group (R-TME: 5 [1-8] cm versus ST-TME: 6 [4-10] $\mathrm{cm} ; P=0.119$ ).

Tables 2 and 3 summarize the comparisons made in terms of operative parameters and postoperative outcomes. The mean operative time was significantly higher in the R-TME group (R-TME: 395 [289-771] minutes versus ST-TME: 242 [191-377] minutes; $P<0.001)$; however, the proportion of patients $(\mathrm{n}=1,4.3 \%)$ in the R-TME group was less than that in the ST-TME group $(\mathrm{n}=4,21.0 \% ; P=0.158)$. The postoperative morbidity was similar between both groups, in which only one patient from each group had a complication grade that

Table 2 Operative parameters

\begin{tabular}{llll}
\hline & ST-TME & R-TME & P-value \\
\hline $\begin{array}{l}\text { Median operative } \\
\text { time, minutes (range) }\end{array}$ & $242(19 \mid-377)$ & $395(289-77 \mid)$ & $<0.00$ I \\
Conversion, n (\%) & & & \\
$\quad$ Narrow pelvis & $0(0.0)$ & $\mathrm{I}(4.3)$ & 0.158 \\
Bulky tumor & $0(0.0)$ & $0(0.0)$ & \\
Hemorrhage & $2(10.5)$ & $0(0.0)$ & \\
Others & $2(10.5)$ & $0(0.0)$ & \\
\hline
\end{tabular}

Abbreviations: R-TME, robotic total mesorectal excision; ST-TME, standard laparoscopic total mesorectal excision.
Table 3 Postoperative outcomes

\begin{tabular}{llll}
\hline & ST-TME & R-TME & P-value \\
\hline $\begin{array}{l}\text { Median duration of parenteral opioid } \\
\text { use, days (range) }\end{array}$ & $6(3 \mathrm{I}-3)$ & $2(\mathrm{I}-3)$ & 0.349 \\
$\begin{array}{l}\text { Morbidity, n (\%) } \\
\text { Grade of complications, n (\%) }\end{array}$ & $5(2 \mathrm{I} .7)$ & 0.504 \\
I & $2(10.5)$ & $\mathrm{I}(4.3)$ & 0.732 \\
II & $3(15.8)$ & $3(13.0)$ & \\
III & $0(0.0)$ & $\mathrm{I}(4.3)$ & \\
IV & $\mathrm{I}(5.3)$ & $0(0.0)$ & \\
Overall & $6(31.6)$ & $5(21.6)$ & \\
Reoperation within 30 days, n (\%) & & & \\
Bleeding & $0(0.0)$ & $\mathrm{I}(4.3)$ & $\mathrm{NS}$ \\
Anastomotic leak & $0(0.0)$ & $0(0.0)$ & \\
$\begin{array}{l}\text { Mortality, n (\%) } \\
\text { Median length of stay, }\end{array}$ & $0(0.0)$ & $0(0.0)$ & $\mathrm{NS}$ \\
days (range) & $6(3-15)$ & $7(4-2 \mathrm{I})$ & 0.202 \\
\hline
\end{tabular}

Note: Complications were graded using the Clavien-Dindo classification. Abbreviations: NS, not significant; R-TME, robotic total mesorectal excision; ST-TME, standard laparoscopic total mesorectal excision.

was more than II. One patient (4.3\%) in the R-TME group required a reoperation due to secondary hemorrhage, while another in the ST-TME group developed a non-ST segment elevation myocardial infarction on postoperative day 4 . The median durations of parenteral opioids used were 3 and 2 days for ST-TME and R-TME, respectively, with a range of 1 to 3 days each $(P=0.349)$. The median length of stay in the hospital for patients who underwent R-TME was 5 days compared to 6 days in the ST-TME group $(P=0.202)$. No mortality was recorded in our series.

Table 4 Distribution of TNM stages

\begin{tabular}{llll}
\hline & ST-TME & R-TME & P-value \\
\hline Tumor stage & & & \\
T0 & $2(10.5)$ & $3(13.0)$ & 0.740 \\
TI & $0(0.0)$ & $0(0.0)$ & \\
T2 & $8(42.1)$ & $6(26.1)$ & \\
T3 & $9(47.4)$ & I $(56.5)$ & \\
T4 & $0(0.0)$ & I $(4.3)$ & \\
Nodal stage & & & \\
N0 & I3 $(68.4)$ & II $(47.8)$ & 0.229 \\
NI & $4(21.1)$ & $4(17.4)$ & \\
N2 & $2(10.5)$ & $8(34.8)$ & \\
Metastatic disease & I (5.3) & $2(8.7)$ & 1.00 \\
Overall stage & & & \\
0 & $2(10.5)$ & $2(8.7)$ & 0.639 \\
I & $7(36.8)$ & $4(17.4)$ & \\
II & $4(2 I .1)$ & $5(2 I .7)$ & \\
III & $5(26.3)$ & I0 (43.5) & \\
IV & I (5.3) & $2(8.7)$ & \\
\hline
\end{tabular}

Notes: The overall stages were determined using the American Joint Committee of Cancer staging. Values are presented as number (\%).

Abbreviations: R-TME, robotic total mesorectal excision; ST-TME, standard laparoscopic total mesorectal excision. 
Table 5 Histopathological assessment

\begin{tabular}{|c|c|c|c|}
\hline & ST-TME (\%) & R-TME (\%) & $P$-value \\
\hline $\begin{array}{l}\text { Completeness of TME } \\
\text { achieved }\end{array}$ & I 8 (94.7) & $23(100.0)$ & 0.452 \\
\hline Distal margin involvement & $0(0.0)$ & I (4.3) & NS \\
\hline Radial margin involvement & I (5.3) & I (4.3) & NS \\
\hline $\begin{array}{l}\text { Median number of lymph } \\
\text { nodes harvested (range) }\end{array}$ & $14(2-39)$ & $14(4-24)$ & 0.323 \\
\hline
\end{tabular}

Abbreviations: NS, not significant; R-TME, robotic TME; ST-TME, standard laparoscopic TME; TME, total mesorectal excision.

Tables 4 and 5 record the distribution of the TNM stages and the histopathological assessment, respectively. There were no significant differences seen between the groups in terms of disease stage, completeness of TME, number of lymph nodes harvested, and distal and circumferential margin positivity. The mean number of lymph nodes harvested was 18 (two to 39) in the ST-TME group and 14 (four to 24) in the R-TME group $(P=0.323)$. Both groups had one patient with a positive circumferential margin (defined as histological evidence of tumor presence at the resection margin), while the R-TME group had one patient $(4.3 \%)$ with a positive distal margin (defined as histological evidence of tumor presence at the resection margin) $(P=1.00)$. The completeness of the TME was $94.7 \%$ and $100 \%$ in the ST-TME and R-TME, respectively $(P=0.452)$.

\section{Discussion}

Laparoscopic colorectal surgery has been established by several studies to be consistent with the benefits of minimally invasive surgery observed in other surgical disciplines, while retaining comparable oncological outcomes to conventional open surgery. ${ }^{2-4}$ However, the inherent technical limitations of laparoscopy, such as the assistant-dependent camera platform, two-dimensional optics, limited instrumental dexterity, and physiological tremors can theoretically limit the purported outcomes for laparoscopic rectal surgeries in isolation due to the confined pelvic compartment in which TME is being performed. This may explain the findings in the CLASICC trial, which reported higher conversion rates and circumferential margin positivity associated with laparoscopic rectal surgeries. $^{2}$

Robotic surgery overcomes such technical limitations of conventional laparoscopic surgery, as it eliminates human factors such as surgeon fatigue and physiological tremors while facilitating greater maneuverability afforded by its EndoWrist $^{\circledR}$ (Intuitive Surgical, Inc.) feature and a stable three-dimensional camera platform. ${ }^{11-13}$ Indeed, the successful implementation of robotic laparoscopic surgery in urological surgeries involving the deep pelvis, such as radical prostatectomy, has prompted the potential utility of employing it in rectal surgeries as well.

In our study, we found R-TME to be associated with lower rates of conversion and postoperative complications compared to ST-TME. This could be attributed to a possibly shorter learning curve for R-TME compared to that for ST-TME, as reported by a recent study. ${ }^{24}$ Furthermore, the fact that $\mathrm{R}-\mathrm{TME}$ is typically performed by surgeons who already have considerable experience in ST-TME may further contribute to greater ease in the transition to the use of R-TME as compared to the initial transition from conventional open surgery to ST-TME. Notwithstanding these arguments, this may well reflect the inherent advantage of R-TME over ST-TME due to the aforementioned features the former possesses.

Median operative time was found to be significantly longer in R-TME, a finding consistent with most other studies ${ }^{13,14,17,18}$ comparing R-TME with ST-TME. While this may serve as a drawback in the use of R-TME, it should be recognized that robotic surgery experience and technology are still in relatively early stages and, with increasing surgical experience, the duration of robotic surgeries may decrease further in the future.

In terms of oncologic outcomes, R-TME and ST-TME were both found to be safe and comparable in terms of completeness of TME, adequacy of resection margins, and the extent of lymph node clearance. However, no studies to date have demonstrated any comparisons in survival and recurrence, and further studies should look into the long-term outcomes between the two modalities.

The small sample size of our study is a limitation, but provides a platform for trend observation and future studies. By having a small number of surgeons within a single institution involved in our study, we limit the amount of heterogeneity that can potentially skew results if present. This is also the first study to report local experience and data with respect to the comparison between R-TME and ST-TME. While limited by its retrospective nature and hence possible selection bias, it can be noted that there was no significant difference in important patient characteristics such as age, sex, ASA score, and neoadjuvant chemotherapy between the two groups. The performance of a randomized controlled trial would be ideal, but it may not be practical given the high costs involved in robotic surgery and that a substantial number of patients is required to demonstrate a small difference. While difficult to qualify, the advancements made in imaging techniques and robotic systems within our study time frame should be taken into account for meaningful interpretation of the study results. 
The cost-effectiveness of R-TME is an important area not addressed in this study. Further studies are essential for examining the feasibility of its usage on a wider basis, despite its potential advantages over ST-TME. Studies have attempted to qualify this aspect of robotic surgery, but they lack reproducibility due to the wide variation of procedural costs between different institutions in different countries with different health care delivery systems. ${ }^{19,25}$

Delving further, subgroups of patients who may particularly benefit from R-TME compared to ST-TME may be identified based on specific desired outcomes. For instance, given the findings from the CLASICC trial, that higher rates of male sexual dysfunction were reported in those undergoing laparoscopic rectal surgery compared to conventional open surgery, R-TME could therefore play a role in improving outcomes of genitourinary function in men while preserving the established benefits of laparoscopic surgery over conventional open surgery. ${ }^{2}$ The COREAN and ROLARR trials are investigating these outcomes, and their results would give us a better platform for comparison between these two modes of treatment. ${ }^{20,26}$

\section{Conclusion}

R-TME has comparable oncologic outcomes with ST-TME and has the advantage of lower rates of conversion and postoperative complications. However, it is also associated with a longer operative time. Given the higher costs of R-TME, further studies are necessary to better define its role in the treatment of rectal cancers.

\section{Disclosure}

The authors report no conflicts of interest in this work.

\section{References}

1. Saclarides TJ, Ko ST, Airan M, Dillon C, Franklin J. Laparoscopic removal of a large colonic lipoma. Report of a case. Dis Colon Rectum. 1991;34(11):1027-1029.

2. Guillou PJ, Quirke P, Thorpe H, et al; MRC CLASICC trial group. Short-term endpoints of conventional versus laparoscopic-assisted surgery in patients with colorectal cancer (MRC CLASICC trial): multicentre, randomised controlled trial. Lancet. 2005;365(9472): 1718-1726.

3. Kennedy GD, Heise C, Rajamanickam V, Harms B, Foley EF. Laparoscopy decreases postoperative complication rates after abdominal colectomy: results from the national surgical quality improvement program. Ann Surg. 2009;249(4):596-601.

4. Lacy AM, García-Valdecasas JC, Delgado S, et al. Laparoscopy-assisted colectomy versus open colectomy for treatment of non-metastatic colon cancer: a randomised trial. Lancet. 2002;359(9325):2224-2229.

5. Ceelen W, Pattyn P. Total mesorectal excision in the treatment of rectal cancer: a review. Acta Chir Belg. 2000;100(3):94-99.
6. Goldberg S, Klas JV. Total mesorectal excision in the treatment of rectal cancer: a view from the USA. Semin Surg Oncol. 1998;15(2):87-90.

7. Kapiteijn E, van De Velde CJ. European trials with total mesorectal excision. Semin Surg Oncol. 2000;19(4):350-357.

8. MacFarlane JK, Ryall RD, Heald RJ. Mesorectal excision for rectal cancer. Lancet. 1993;341(8843):457-460.

9. Murty M, Enker WE, Martz J. Current status of total mesorectal excision and autonomic nerve preservation in rectal cancer. Semin Surg Oncol. 2000;19(4):321-328.

10. Heald RJ, Husband EM, Ryall RD. The mesorectum in rectal cancer surgery - the clue to pelvic recurrence? Br J Surg. 1982;69(10): 613-616.

11. Mirnezami AH, Mirnezami R, Venkatasubramaniam AK, Chandrakumaran K, Cecil TD, Moran BJ. Robotic colorectal surgery: hype or new hope? A systematic review of robotics in colorectal surgery. Colorectal Dis. 2010;12(11):1084-1093.

12. Pigazzi A, Garcia-Aguilar J. Robotic colorectal surgery: for whom and for what? Dis Colon Rectum. 2010;53(7):969-970.

13. Delaney CP, Lynch AC, Senagore AJ, Fazio VW. Comparison of robotically performed and traditional laparoscopic colorectal surgery. Dis Colon Rectum. 2003;46(12):1633-1639.

14. Memon S, Heriot AG, Murphy DG, Bressel M, Lynch AC. Robotic versus laparoscopic proctectomy for rectal cancer: a meta-analysis. Ann Surg Oncol. 2012;19(7):2095-2101.

15. Baik SH, Kwon HY, Kim JS, et al. Robotic versus laparoscopic low anterior resection of rectal cancer: short-term outcome of a prospective comparative study. Ann Surg Oncol. 2009;16(6):1480-1487.

16. Bianchi PP, Ceriani C, Locatelli A, et al. Robotic versus laparoscopic total mesorectal excision for rectal cancer: a comparative analysis of oncological safety and short-term outcomes. Surg Endosc. 2010;24(11):2888-2894.

17. Kwak JM, Kim SH, Kim J, Son DN, Baek SJ, Cho JS. Robotic vs laparoscopic resection of rectal cancer: short-term outcomes of a casecontrol study. Dis Colon Rectum. 2011;54(2):151-156.

18. Park JS, Choi GS, Lim KH, Jang YS, Jun SH. Robotic-assisted versus laparoscopic surgery for low rectal cancer: case-matched analysis of short-term outcomes. Ann Surg Oncol. 2010;17(12):3195-3202.

19. Baek JH, Pastor C, Pigazzi A. Robotic and laparoscopic total mesorectal excision for rectal cancer: a case-matched study. Surg Endosc. 2011;25(2):521-525.

20. Collinson FJ, Jayne DG, Pigazzi A, et al. An international, multicentre, prospective, randomised, controlled, unblinded, parallel-group trial of robotic-assisted versus standard laparoscopic surgery for the curative treatment of rectal cancer. Int J Colorectal Dis. 2012;27(2):233-241.

21. Alliance for Clinical Trials in Oncology. Laparoscopic-Assisted Resection or Open Resection in Treating Patients With Rectal Cancer. Available from: http://clinicaltrials.gov/show/NCT00726622. NLM identifier: NCT00726622. Accessed July 1, 2013.

22. Shawki S, Bashankaev B, Denoya P, Seo C, Weiss EG, Wexner SD. What is the definition of "conversion" in laparoscopic colorectal surgery? Surg Endosc. 2009;23(10):2321-2326.

23. Clavien PA, Barkun J, de Oliveira ML, et al. The Clavien-Dindo classification of surgical complications: five-year experience. Ann Surg. 2009;250(2):187-196.

24. Park IJ, Choi GS, Lim KH, Kang BM, Jun SH. Multidimensional analysis of the learning curve for laparoscopic resection in rectal cancer. J Gastrointest Surg. 2009;13(2):275-281.

25. Hottenrott C. Robotic versus laparoscopic surgery for rectal cancer and cost-effectiveness analysis. Surg Endosc. 2011;25(12):3954-3956; author reply 3957-3958.

26. National Cancer Center, Korea. Randomized Prospective Trial for Laparoscopic vs Open Resection for Rectal Cancer (CTS-179). Available from: http://clinicaltrials.gov/show/NCT00470951. NLM identifier: NCT00470951. Accessed July 1, 2013. 
Robotic Surgery: Research and Reviews

Dovepress

\section{Publish your work in this journal}

Robotic Surgery: Research and Reviews is an international, peer reviewed, open access, online journal publishing original research, commentaries, reports, and reviews on the theory, use and application of robotics in surgical interventions. Articles on the use of supervisory-controlled robotic systems, telesurgical devices, and shared-control systems are

invited. The manuscript management system is completely online and includes a very quick and fair peer review system, which is all easy to use. Visit http://www.dovepress.com/testimonials.php to read real quotes from published authors.

Submit your manuscript here: http://www.dovepress.com/robotic-surgery-research-and-reviews-journal 